\title{
Perturbation Solutions for Hagen-Poiseuille Flow and Heat Transfer of Third-Grade Fluid with Temperature-Dependent Viscosities and Internal Heat Generation
}

\author{
B. Y. Ogunmola, A. T. Akinshilo, and M. G. Sobamowo \\ Department of Mechanical Engineering, University of Lagos, Akoka-Yaba, Lagos, Nigeria \\ Correspondence should be addressed to M. G. Sobamowo; mikegbeminiyiprof@yahoo.com
}

Received 30 June 2016; Accepted 6 September 2016

Academic Editor: Giuseppe Carbone

Copyright (C) 2016 B. Y. Ogunmola et al. This is an open access article distributed under the Creative Commons Attribution License, which permits unrestricted use, distribution, and reproduction in any medium, provided the original work is properly cited.

\begin{abstract}
Regular perturbation technique is applied to analyze the fluid flow and heat transfer in a pipe containing third-grade fluid with temperature-dependent viscosities and heat generation under slip and no slip conditions. The obtained approximate solutions were used to investigate the effects of slip on the heat transfer characteristics of the laminar flow in a pipe under Reynolds's and Vogel's temperature-dependent viscosities. Also, the effects of parameters such as variable viscosity, non-Newtonian parameter, viscous dissipation, and pressure gradient at various values were established. The results of this work were compared with the numerical results found in literature and good agreements were established. The results can be used to advance the analysis and study of the behavior of third-grade fluid flow and steady state heat transfer processes such as those found in coal slurries, polymer solutions, textiles, ceramics, catalytic reactors, and oil recovery applications.
\end{abstract}

\section{Introduction}

The third-grade fluid being a subclass of non-Newtonian fluid whose viscosity varies based on the applied force is a favored fluid due to the exciting phenomena it captures such as the shear thinning and thickening effects. The mathematical model of a third-grade fluid represents a more realistic description of the behavior of non-Newtonian fluids. The model also represents a further attempt towards the study of the flow structure of non-Newtonian fluids. Consequently, considerable interest has been shown in the third-grade fluid over the past few decades by various researchers due to its potential application in industry and technology. It should be stated that the governing equations for the third-grade fluid model are nonlinear and much more complicated than those of Newtonian fluids. They require additional boundary conditions to obtain a physically meaningful solution. This issue has been discussed in detail by researchers [1-23] who made a complete thermodynamical analysis of a third-grade fluid and derived the restriction on the stress constitutive equation. They investigated some stability characteristics of third-grade fluids and showed that they exhibit features different from those of Newtonian and second-grade fluids. In an attempt to improve the characteristics of the viscoelastic properties of the fluid, the stability of the third-grade fluid model was studied by Fosdick and Rajagopal [1] while Majhi and Nair [2] investigated the effects of stenotic geometry and the non-Newtonian parameter of the third-grade fluid on the resistive impedance and wall shearing stress. Their results were compared with similar study submitted by Massoudi and Christie [3] who presented the numerical solutions on the effects of variable viscosity and viscous dissipation on the flow of third-grade fluid in a pipe using the finite difference method. Yürüsoy and Pakdemirli [4] developed approximate analytical solution for the flow of the third-grade fluid in a pipe using constant viscosity model and variable viscosity models under no slip condition while Vajravelu et al. [5] presented a numerical solution for the thirdgrade fluid flows between rotating cylinder using Schauder theory and perturbation technique. The fluctuating flow of a magnetohydrodynamic rotational flow of the third-grade fluid on a porous plate was studied by Hayat et al. [6]. The similarity solutions to boundary layer equation for the thirdgrade fluid were developed by Muhammet [7] using the 
special coordinate system generated by the potential flow. The steady flow analysis of the third-grade fluid between circular concentric cylinders with heat transfer was presented by Yürüsoy [8]. In the study, the pipe temperature is assumed to be higher than fluid temperature. Also, Pakdemirli and Yilbas [9] developed approximate analytical solution of nonNewtonian fluid using Vogel's viscosity model and entropy generation in a pipe. The steady flow of a third-grade fluid past a porous horizontal plate with partial slip was investigated by Sajid et al. [10]. Ellahi et al. [11] used implicit finite difference method to analyze the unsteady free convective flow of a third-grade fluid past an infinite vertical plate when uniform suction is applied at the plate. Jayeoba and Okoya [12] presented the analytical approximate solution to determine the temperature fields for steady flow of a thirdgrade fluid in a pipe with models of viscosities including a heat generation term for the no slip boundary condition. Most of the studies previously carried out on the thirdgrade fluid are limited to no slip flow condition which is a simplified method of predicting the actual behavior of the fluid in various applications. In practice, most problems of fluid flow exist as either partial slip or slip condition, Hence, in this work, regular perturbation method is used to develop approximate analytical solutions that are used to investigate the effects of slip on the heat transfer analysis in pipe flow of third-grade fluid with temperature-dependent viscosities and internal heat generation.

\section{Model Development and Analytical Solution}

Consider the flow of a third-grade fluid in a pipe as shown in Figure 1. The momentum and temperature equations with incorporated source term are given by $[3,4,13]$

$$
\begin{aligned}
& \frac{1}{r} \frac{d}{d r}\left(r\left(2 \propto_{1}+\propto_{2}\right)\left[\frac{d w}{d r}\right]^{2}\right)=\frac{\partial p}{\partial r}, \\
& 0=\frac{\partial p}{\partial \emptyset}, \\
& \frac{1}{r} \frac{d}{d r}\left(r \mu \frac{d w}{d r}\right)+\frac{1}{r} \frac{d}{d r}\left(2 r \beta_{3}\left[\frac{d w}{d r}\right]^{3}\right)=\frac{\partial p}{\partial z}, \\
& k\left(\frac{1}{r} \frac{d}{d r}\left(r \frac{d T}{d r}\right)\right)+\mu\left(\frac{d w}{d r}\right)^{2}+2 \beta_{3}\left(\frac{d w}{d r}\right)^{4} \\
& \quad+Q C_{0}\left(T-T_{0}\right)=0 .
\end{aligned}
$$

The slip condition at the pipe wall can be introduced in terms of shear stress

$$
\begin{aligned}
& w(R)=\left.\gamma\left[\frac{d w}{d r}+\frac{2 \beta_{3}}{\mu}\left(\frac{d w}{d r}\right)^{3}\right]\right|_{r=R}, \\
& h\left(T(R)-T_{s}\right) \\
& =-\left.k\left[\frac{d \theta}{d r}+\mu\left(\frac{d w}{d r}\right)^{2}+2 \beta_{3}\left(\frac{d w}{d r}\right)^{4}\right]\right|_{r=R},
\end{aligned}
$$

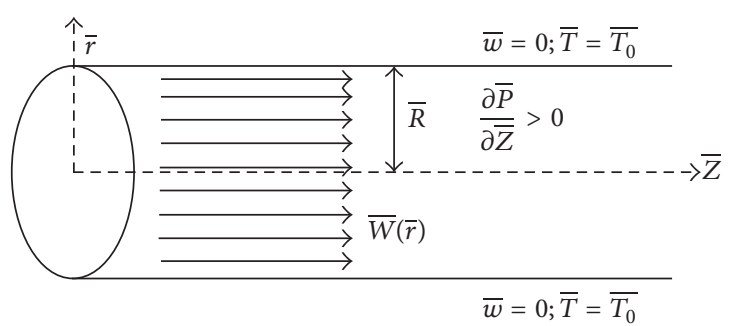

Figure 1: Physical model and coordinate system [12].

which makes the boundary condition nonlinear. Linearizing the boundary condition at the wall gives a corresponding linear boundary condition at the wall under slip condition as

$$
\begin{aligned}
w(1) & =\left.\gamma_{l}\left[\frac{d w}{d r}\right]\right|_{r=R}, \\
h\left(T(R)-T_{s}\right) & =-\left.k_{l}\left[\frac{d \theta}{d r}\right]\right|_{r=R} .
\end{aligned}
$$

At the center of the pipe, the boundary condition is given as

$$
\begin{aligned}
& \left.\frac{d w}{d r}\right|_{r=0}=0 \\
& \left.\frac{d T}{d r}\right|_{r=0}=0 .
\end{aligned}
$$

The boundary conditions under slip condition are given as

$$
\begin{aligned}
w(R) & =\left.A_{P} \frac{d w}{d r}\right|_{r=R}, \\
h\left(T(R)-T_{s}\right) & =-\left.k_{l}\left[\frac{d T}{d r}\right]\right|_{r=R} .
\end{aligned}
$$

With the dimensionless parameters as stated in the nomenclature, the dimensionless equations (leaving out the bars on the equations) for (3) and (4) are given as

$$
\begin{aligned}
& \frac{d \mu}{d r} \frac{d w}{d r}+\frac{\mu}{r}\left(\frac{d w}{d r}+\frac{r d^{2} w}{d r^{2}}\right) \\
& +\frac{\Lambda}{r}\left(\frac{d w}{d r}\right)^{2}\left(\frac{d \omega}{d r}+\frac{3 r d^{2} w}{d r^{2}}\right)=C, \\
& \frac{d^{2} \theta}{d r^{2}}+\frac{1}{r} \frac{d \theta}{d r}+\Gamma\left(\frac{d w}{d r}\right)^{2}\left(\mu+\Lambda\left(\frac{d w}{d r}\right)^{2}\right)+\delta \theta=0 .
\end{aligned}
$$

And the dimensionless boundary conditions under slip condition are given as

$$
\begin{aligned}
& \frac{d w}{d r}(0)=0, \\
& \frac{d \theta}{d r}(0)=0
\end{aligned}
$$




$$
\begin{aligned}
& w(1)=\left.A_{P} \frac{d w}{d r}\right|_{r=1 / D}, \\
& \theta(1)=-\left.\frac{1}{B i} \frac{d \theta}{d r}\right|_{r=1 / D},
\end{aligned}
$$

where $D=1+8 \mathrm{Kn}$ and $A_{p}=-2 \mathrm{Kn}$.

In this work, two equations of temperature-dependent viscosity are used, Reynolds and Vogel's models.

$$
\begin{array}{ll}
\mu(T)=\mu_{0} e^{\left(-M\left(T-T_{0}\right)\right)} & \text { Reynolds model } \\
\mu(T)=\mu_{0} e^{\left(a /\left(b-T_{0}\right)\right)} & \text { Vogel's model. }
\end{array}
$$

Reynolds model is a model of viscosity varying constantly with temperature while Vogel's model of viscosity is varying exponentially with temperature. The nondimensionalized form of both models yields

$$
\begin{aligned}
& \mu=e(-Y \theta) \quad \text { Reynolds model } \\
& \mu=e^{\left(A /(B-\theta)-T_{0}\right)} \quad \text { Vogel's model. }
\end{aligned}
$$

2.1. Reynolds Model of Viscosity. The regular perturbation technique is used to determine the approximate analytical solution for the Reynolds viscosity model.

Taking Maclaurin's series, (13a) takes the form

$$
\mu=\exp (-Y \theta)=1-Y \theta+O\left(Y^{2}\right)
$$

Series solutions of the equation of the velocity and temperature may be obtained by using perturbation method taking $\epsilon$ as the perturbation parameter. Solutions are obtained in the following form:

$$
\begin{aligned}
& w=w_{0}+\epsilon w_{1}+O\left(\epsilon^{2}\right), \\
& \theta=\theta_{0}+\epsilon \theta_{1}+O\left(\epsilon^{2}\right) .
\end{aligned}
$$

Substituting (15) into (9) and changing parameters into terms yield

$$
\begin{aligned}
& O\left(\epsilon^{0}\right): r \frac{d^{2} w_{0}}{d r^{2}}+\frac{d w_{0}}{d r}=C r \\
& O\left(\epsilon^{1}\right): \frac{d w_{1}}{d r}+r \frac{d^{2} w_{1}}{d r^{2}}-P \theta_{0} \frac{d w_{0}}{d r}-P \theta_{0} \frac{d^{2} w_{0}}{d r^{2}} \\
& \quad-\operatorname{Pr} \frac{d w_{0}}{d r} \frac{d \theta_{0}}{d r}=-M\left(\frac{d w_{0}}{d r}\right)^{2}\left(\frac{d w_{0}}{d r}+3 r \frac{d^{2} w_{0}}{d r^{2}}\right) \\
& \quad+C r
\end{aligned}
$$

Also, substituting (14) and (15) into (10) and changing parameters into terms yield

$$
\begin{aligned}
& O\left(\epsilon^{0}\right): r \frac{d^{2} \theta_{0}}{d r^{2}}+\frac{d \theta_{0}}{d r}+\frac{d \theta_{0}}{d r}+\Gamma r\left(\frac{d w_{0}}{d r}\right)^{2}=0, \\
& \theta\left(\epsilon^{1}\right): r \frac{d^{2} \theta_{1}}{d r^{2}}+\frac{d \theta_{0}}{d r}+\frac{d \theta_{1}}{d r}+\Gamma r\left(\frac{d w_{0}}{d r}\right)^{2} \\
& \cdot\left(M\left(\frac{d w_{0}}{d r}\right)^{2}-P \theta_{0}\right)+2 \Gamma r \frac{d w_{0}}{d r} \frac{d w_{1}}{d r} \\
& +r N \theta_{0}=0 .
\end{aligned}
$$

The boundary conditions for the leading order equation are

$$
\begin{aligned}
\frac{d w_{0}}{d r}(0) & =\frac{d \theta_{0}}{d r}(0)=0, \\
w_{0}(1) & =\left.A_{P} \frac{d w_{0}}{d r}\right|_{r=1 / D}, \\
\theta_{0}(1) & =-\left.\frac{1}{B i} \frac{d \theta_{0}}{d r}\right|_{r=1 / D},
\end{aligned}
$$

where $\Lambda=\epsilon M, \delta=\epsilon N, \gamma=\epsilon P$.

With the boundary conditions, it could be easily shown that (16) and (18) are given as

$$
\begin{aligned}
& w_{0}=\frac{C}{4}\left[r^{2}+\frac{2 A_{p}}{D}-\frac{1}{D^{2}}\right] \\
& \theta_{0}=\frac{\Gamma C^{2}}{64}\left[1-r^{4}+\frac{4}{B i D^{3}}\right] .
\end{aligned}
$$

Also, for the first-order equation, the boundary conditions are given as

$$
\begin{aligned}
\frac{d w_{1}}{d r}(0) & =\frac{d \theta_{1}}{d r}(0)=0, \\
w_{1}(1) & =\left.A_{P} \frac{d w_{1}}{d r}\right|_{r=1 / D}, \\
\theta_{1}(1) & =\frac{-1}{B i} \frac{d \theta_{1}}{d r},
\end{aligned}
$$

where $D=1+8 \mathrm{Kn}$ and $A_{p}=-2 \mathrm{Kn}$.

Substituting the corresponding terms from the solutions in (21) into (17) and (19) and integrating the resulting 
equations two times successively, with the applications of the boundary conditions in (22) yields

$$
\begin{aligned}
w_{1} & =\frac{A_{P} P \Gamma C^{3}}{64}\left[\frac{1}{2 D}-\frac{1}{6 D^{5}}+\frac{2}{B i D^{4}}\right]-\frac{A_{P} P \Gamma C^{3}}{192 D^{2}} \\
& -\frac{A_{P} M C^{5}}{8 D^{3}}-\frac{P \Gamma C^{3}}{1152}+\frac{P \Gamma C^{3}}{64}\left[\frac{r^{2}}{4}-\frac{r^{6}}{36}+\frac{r^{2}}{B i D^{3}}\right. \\
& \left.-\frac{1}{4 D^{2}}+\frac{1}{36 D^{6}}-\frac{1}{B i D^{5}}\right]-\frac{M C^{3}}{32}\left[r^{4}-\frac{1}{D^{4}}\right] \\
\theta_{1} & =\frac{M \Gamma C^{4}}{96 B i D^{5}}-\frac{P \Gamma^{2} C^{4}}{256 B i}\left[\frac{1}{4 D^{3}}-\frac{1}{8 D^{7}}+\frac{1}{B i D^{6}}\right] \\
& +\frac{P \Gamma^{2} C^{4}}{46 B i}\left[\frac{1}{8 D^{3}}-\frac{1}{48 D^{7}}+\frac{1}{2 B i D^{6}}\right]-\frac{P \Gamma^{2} C^{4}}{153 B i D^{7}} \\
& -\frac{M C^{4} \Gamma}{48 B i d^{5}}+\frac{\Gamma C^{2} N}{64 B i}\left[\frac{1}{2 D}-\frac{1}{6 D^{5}}+\frac{2}{B i D^{4}}\right] \\
& -\frac{M \Gamma C^{4}}{576}\left[r^{4}-\frac{1}{D^{6}}\right]+\frac{P \Gamma^{2} C^{4}}{256}\left[\frac{r^{4}}{16}-\frac{r^{8}}{64}\right. \\
& \left.+\frac{r^{4}}{4 B i D^{3}}-\frac{1}{16 D^{4}}+\frac{1}{64 D^{8}}-\frac{1}{14 B i D^{7}}\right] \\
& -\frac{P \Gamma^{2} C^{4}}{64}\left[\frac{r^{4}}{32}-\frac{r^{8}}{384}+\frac{r^{3}}{6 B i D^{3}}-\frac{1}{32 D^{4}}+\frac{1}{384 D^{8}}\right. \\
& \left.-\frac{1}{6 B i D^{6}}\right]+\frac{P \Gamma^{2} C^{4}}{12288}\left[r^{8}-\frac{1}{D^{8}}\right]+\frac{M C^{4} \Gamma}{288}\left[r^{6}\right. \\
& 6 \frac{r^{2} N}{4}-\frac{r^{6}}{36}+\frac{r^{2}}{B i D^{3}}-\frac{1}{4 D}+\frac{1}{36 D^{6}} \\
& \\
& \\
&
\end{aligned}
$$

Substituting (21) and (23) into (15) the series solution at the order of expansion and then changing terms back to original parameters finally give

$$
\begin{aligned}
w & =\frac{C}{4}\left[r^{2}+\frac{2 A_{P}}{D}-\frac{1}{D^{2}}\right]+\frac{\gamma A_{P} \Gamma C^{3}}{64}\left[\frac{1}{2 D}-\frac{1}{6 D^{5}}\right. \\
& \left.+\frac{2}{B i D^{4}}\right]-\frac{\gamma A_{P} \Gamma C^{3}}{192 D^{5}}-\frac{\Lambda A_{P} C^{3}}{8 D^{3}}+\frac{\gamma \Gamma C^{3}}{64}\left[\frac{r^{2}}{4}\right. \\
& \left.-\frac{r^{6}}{36}+\frac{r^{2}}{B i D^{3}}-\frac{1}{4 D^{2}}+\frac{1}{36 D^{6}}-\frac{1}{B i D^{5}}\right] \\
& -\frac{\gamma \Gamma C^{3}}{1152}\left[r^{6}-\frac{1}{D^{6}}\right]-\frac{\Lambda C^{3}}{32}\left[r^{6}-\frac{1}{D^{6}}\right],
\end{aligned}
$$

$$
\begin{aligned}
\theta & =\frac{\Gamma C^{2}}{64}\left[1-r^{4}+\frac{4}{B i D^{3}}\right]+\frac{\Lambda \Gamma C^{4}}{96 B i D^{5}}-\frac{\gamma \Gamma^{2} C^{4}}{256 B i}\left[\frac{1}{4 D^{3}}\right. \\
& \left.-\frac{1}{8 D^{7}}+\frac{1}{B i D^{6}}\right]+\frac{\gamma \Gamma^{2} C^{4}}{64 B i}\left[\frac{1}{8 D^{3}}-\frac{1}{48 D^{7}}\right. \\
& \left.+\frac{1}{2 B i D^{5}}\right]-\frac{\gamma \Gamma^{2} C^{4}}{1536 B i D^{7}}-\frac{\Lambda C^{4} \Gamma}{48 B i D^{5}}+\frac{\partial \Gamma C^{2}}{64 B i}\left[\frac{1}{2 D}\right. \\
& \left.-\frac{1}{6 D^{5}}+\frac{2}{B i D^{4}}\right]-\frac{\Lambda \Gamma C^{4}}{576}\left[r^{6}-\frac{1}{D^{6}}\right] \\
& +\frac{Y \Gamma^{2} C^{4}}{256}\left[\frac{r^{4}}{16}-\frac{r^{8}}{64}+\frac{r^{4}}{4 B i D^{3}}-\frac{1}{16 D^{4}}+\frac{1}{64 D^{8}}\right. \\
& \left.-\frac{1}{14 B i D^{7}}\right]-\frac{Y \Gamma^{2} C^{4}}{64}\left[\frac{r^{4}}{32}-\frac{r^{8}}{384}+\frac{r^{3}}{6 B i D^{3}}\right. \\
& \left.-\frac{1}{32 D^{4}}+\frac{1}{384 D^{8}}-\frac{1}{6 B i D^{6}}\right]+\frac{P \Gamma^{2} C^{4}}{12288}\left[r^{8}-\frac{1}{D^{8}}\right] \\
& +\frac{\Lambda C^{4} \Gamma}{288}\left[r^{6}-\frac{1}{D^{6}}\right]-\frac{\partial \Gamma C^{2}}{64}\left[\frac{r^{2}}{4}-\frac{r^{6}}{36}+\frac{r^{2}}{B i D^{3}}\right. \\
& \left.-\frac{1}{4 D}+\frac{1}{36 D^{6}}-\frac{1}{B i D^{5}}\right] .
\end{aligned}
$$

2.2. Vogel's Model of Viscosity. With the aid of Maclaurin series, Vogel's viscosity model can be written as

$$
\begin{aligned}
\mu & =\exp \left(\frac{A}{B}-T_{0}\right)\left(1-\frac{\epsilon A \theta}{B^{2}}+O\left(\epsilon^{2}\right)\right) \\
& =\exp \left(\frac{A}{B}-T_{0}\right)\left(1-\frac{\epsilon A \theta}{B^{2}}\right) .
\end{aligned}
$$

Taking the series solution of the velocity and temperature fields yields the expansion

$$
\begin{aligned}
& w=w+\epsilon w_{1}+O\left(\epsilon^{2}\right), \\
& \theta=\epsilon \theta_{0}+\epsilon^{2} \theta_{1}+O\left(\epsilon^{3}\right) .
\end{aligned}
$$

Therefore substituting (26) and (27) into (9) and changing parameters into term yield

$$
O\left(\epsilon^{0}\right): r \frac{d^{2} w_{0}}{d r^{2}}+\frac{d w_{0}}{d r}=C^{*} r
$$

where $C^{*}=C / \exp \left(A / B-T_{0}\right)$,

$$
\begin{aligned}
O\left(\epsilon^{0}\right) & : r \frac{d^{2} \theta_{0}}{d r^{2}}+\frac{d w_{1}}{d r} \\
= & \frac{A}{B^{2}} \theta_{0}\left(r \frac{d^{2} w_{0}}{d r^{2}}+\frac{d w_{0}}{d r}\right)+\frac{A}{B^{2}} r \frac{d \theta_{0}}{d r} \frac{d w_{0}}{d r} \\
& -\frac{M C^{*}}{C}\left(\frac{d w_{0}}{d r}\right)^{2}\left(3 r \frac{d^{2} w_{0}}{d r^{2}}+\frac{d \omega_{0}}{d r}\right),
\end{aligned}
$$

where $\Lambda=\epsilon M, \delta=\epsilon N, \Gamma=\epsilon Q$. 
Substituting (26), (27), and (28) into (10) and changing parameters into term yield

$$
O\left(\epsilon^{1}\right): r \frac{d^{2} \theta_{0}}{d r^{2}}+\frac{d \theta_{0}}{d r}+Q r\left(\frac{d w_{0}}{d r}\right)^{2} \frac{C}{C^{*}}=0
$$

where $C^{*}=C / \exp \left(A / B-T_{0}\right), \Lambda=\epsilon M, \delta=\epsilon N, \Gamma=\epsilon Q$,

$$
\begin{aligned}
\theta\left(\epsilon^{2}\right): r \frac{d^{2} \theta_{1}}{d r^{2}}+\frac{d \theta_{1}}{d r}= & Q r \frac{C}{C^{*}} \frac{A}{B^{2}} \theta_{0}\left(\frac{d w_{0}}{d r}\right)^{2} \\
& -2 r Q \frac{C}{C^{*}} \frac{d w_{0}}{d r} \frac{d w_{1}}{d r} \\
& -r Q M\left(\frac{d w_{0}}{d r}\right)^{4}-N r \theta_{0}
\end{aligned}
$$

$$
=0 \text {. }
$$

The boundary conditions for the leading order are as follows:

$$
\begin{aligned}
\frac{d w_{0}}{d r}(0) & =\frac{d \theta_{0}}{d r}(0)=0, \\
w_{0}(1) & =\left.A_{P} \frac{d w_{0}}{d r}\right|_{r=1 / D}, \\
\theta_{0}(1) & =-\left.\frac{1}{B i} \frac{d \theta_{0}}{d r}\right|_{r=1 / D} .
\end{aligned}
$$

Using the boundary condition, it could be easily shown that (29) and (30) are given as

$$
\begin{aligned}
& w_{0}=\frac{C^{*}}{4}\left[r^{2}+\frac{2 A_{p}}{D}-\frac{1}{D^{2}}\right], \\
& \theta_{0}=\frac{Q C C^{*}}{64}\left[\frac{4}{D^{3} B i}-r^{4}+\frac{1}{D^{4}}\right] .
\end{aligned}
$$

Also, the first-order boundary condition is as follows:

$$
\begin{aligned}
\frac{d w_{1}}{d r}(0) & =\frac{d \theta_{1}}{d r}(0)=0 \\
w_{1}(1) & =\left.A_{P} \frac{d w_{1}}{d r}\right|_{r=1 / D}, \\
\theta_{1}(1) & =\frac{-1}{B i} \frac{d \theta_{1}}{d r}
\end{aligned}
$$

where $D=1+8 \mathrm{Kn}$ and $A_{p}=-2 \mathrm{Kn}$.
Substituting the corresponding terms from the solutions in (33) and (34) into (30) and (32) and integrating the resulting equations two times successively, with the applications of the boundary conditions in (36) yields

$$
\begin{aligned}
& w_{1}=\frac{A_{P} A Q C C^{* 2}}{64 B^{2}}\left[\frac{2}{D^{4} B i}-\frac{1}{6 D^{5}}+\frac{1}{2 D^{5}}\right] \\
& +\frac{A_{P} A Q C C^{*}}{192 B^{2} D^{5}}+\frac{A_{p} M C^{* 4}}{8 C D^{3}}+\frac{A Q C C^{* 2}}{64 B^{2}}\left(\frac{r^{2}}{D^{3} B i}\right. \\
& \left.-\frac{r^{6}}{36}+\frac{r^{2}}{4 D^{4}}-\frac{1}{D^{5} B i}+\frac{1}{36 D^{6}}-\frac{1}{4 D^{6}}\right) \\
& +\frac{A Q C C^{*}}{1152 B^{2}}\left[r^{6}-\frac{1}{D^{6}}\right]+\frac{M C^{* 4}}{32 C}\left[r^{4}-\frac{1}{D^{4}}\right], \\
& \theta_{1}=\frac{Q^{2} C^{2} C^{*} A}{256 B^{2} \cdot B i}\left[\frac{1}{D^{6} B i}-\frac{1}{8 D^{7}}+\frac{1}{4 D^{4}}\right] \\
& +\frac{A Q^{2} C^{2} C^{* 2}}{64 B^{2} \cdot B i}\left[\frac{1}{2 D^{6} B i}-\frac{1}{48 D^{7}}+\frac{1}{8 D^{7}}\right] \\
& +\frac{A Q^{2} C^{2} C^{*}}{1536 B^{2} \cdot B i D^{7}}+\frac{M C C^{* 4} q}{48 C B i \cdot D^{5}}+\frac{C^{* 4} Q M}{96 B i \cdot D^{5}} \\
& +\frac{\mathrm{QCC}^{*} N}{64 B i}\left[\frac{2}{D^{4} B i}-\frac{1}{6 D^{5}}+\frac{1}{2 D^{5}}\right] \\
& +\frac{Q^{2} C C^{* 2} A}{256 B^{2}}\left[\frac{r^{4}}{4 D^{3} B i}-\frac{r^{8}}{64}+\frac{r^{4}}{16 D^{4}}-\frac{1}{4 D^{7} B i}\right. \\
& \left.+\frac{1}{64 D^{8}}-\frac{1}{16 D^{8}}\right]-\frac{A Q^{2} C^{2} C^{* 2}}{64 B^{2}}\left[\frac{r^{4}}{8 D^{3} B i}-\frac{r^{8}}{384}\right. \\
& \left.+\frac{r^{4}}{32 D^{4}}-\frac{1}{8 D^{7} B i}+\frac{1}{38 D^{8}}-\frac{1}{32 D^{8}}\right] \\
& -\frac{A Q^{2} C^{2} C^{*}}{12288 B^{2}}\left[r^{8}-\frac{1}{D^{8}}\right]-\frac{M C C^{* 4} Q}{288 C}\left[r^{6}-\frac{1}{D^{6}}\right] \\
& -\frac{C^{* 4} Q M}{576}\left[r^{6}-\frac{1}{D^{6}}\right]-\frac{Q C C^{*} N}{64}\left[\frac{r^{2}}{D^{3} B i}-\frac{r^{6}}{36}\right. \\
& \left.+\frac{r^{2}}{4 D^{4}}-\frac{1}{D^{5} B i}+\frac{1}{36 D^{6}}-\frac{1}{4 D^{6}}\right] \text {. }
\end{aligned}
$$

Substituting (34) and (37), (35), and (38) into (27) and (28), the series solution at the order of expansion, and then changing terms back to original parameters finally give

$$
\begin{aligned}
w & =\frac{C^{*}}{4}\left[r^{2}+\frac{2 A_{P}}{D}-\frac{1}{D^{2}}\right]+\frac{A_{P} \Gamma A C C^{* 2}}{64 B^{2}}\left[\frac{2}{D^{4} B i}\right. \\
& \left.-\frac{1}{6 D^{5}}+\frac{1}{2 D^{5}}\right]+\frac{A_{P} \Gamma A C C^{*}}{192 B^{2} D^{5}}+\frac{A_{P} \Lambda C^{* 4}}{8 C D^{3}} \\
& +\frac{A_{P} \Gamma A C C^{* 2}}{64 B^{2}}\left[\frac{r^{2}}{D^{3} B i}-\frac{r^{6}}{36}+\frac{r^{2}}{4 D^{4}}-\frac{1}{D^{5} B i}\right.
\end{aligned}
$$




$$
\begin{aligned}
& \left.+\frac{1}{36 D^{6}}-\frac{1}{4 D^{6}}\right]+\frac{A \Gamma C C^{*}}{1152 B^{2}}\left[r^{6}-\frac{1}{D^{6}}\right] \\
& +\frac{\Lambda C^{* 4}}{32 C}\left[r^{4}-\frac{1}{D^{4}}\right], \\
\theta & =\frac{\Gamma C C^{*}}{64}\left[\frac{4}{D^{3} B i}-r^{4}+\frac{1}{D^{4}}\right]+\frac{\Gamma^{2} C^{2} C^{* 2} A}{256 B^{2} \cdot B i}\left[\frac{1}{D^{6} B i}\right. \\
& \left.+\frac{1}{8 D^{2}}+\frac{1}{4 D^{4}}\right]+\frac{\Gamma^{2} A C^{2} C^{* 2}}{64 B^{2} \cdot B i}\left[\frac{1}{2 D^{6} B i}-\frac{1}{48 D^{7}}\right. \\
& \left.+\frac{1}{8 D^{7}}\right]+\frac{\Gamma^{2} A C^{2} C^{*}}{1536 B^{2} \cdot B i D^{7}}+\frac{\Lambda C^{* 4} \Gamma}{48 B i \cdot D^{5}} \\
& +\frac{C^{* 4} \Gamma \Lambda}{96 B i \cdot D^{5}}+\frac{\Gamma C C^{*} \partial}{64 B i}\left[\frac{2}{D^{4} \cdot B i}-\frac{1}{6 D^{5}}+\frac{1}{2 D^{5}}\right] \\
& +\frac{\Gamma^{2} C^{2} C^{* 2} A}{256 B^{2}}\left[\frac{r^{4}}{4 D^{3} B i}-\frac{r^{8}}{64}+\frac{r^{4}}{16 D^{4}}-\frac{1}{4 D^{7} B i}\right. \\
& \left.+\frac{1}{64 D^{8}}-\frac{1}{16 D^{8}}\right] \frac{\Gamma^{2} A C^{2} C^{* 2}}{1228 B^{2}}\left[r^{8}-\frac{1}{D^{8}}\right] \\
& -\frac{\Lambda C^{* 4}}{288}\left[r^{6}-\frac{1}{D^{6}}\right]-\frac{\Gamma C^{* 4} \Lambda}{576}\left[r^{6}-\frac{1}{D^{6}}\right] \\
& -\frac{\Gamma C C^{*} \partial}{64}\left[\frac{r^{2}}{D^{3} B i}-\frac{r^{6}}{36}+\frac{r^{2}}{4 D^{4}}-\frac{1}{D^{5} B i}-\frac{1}{36 D^{6}}\right. \\
& \left.-\frac{1}{4 D^{6}}\right],
\end{aligned}
$$

where

$$
C^{*}=\frac{c}{\exp \left(A / B-T_{0}\right)}
$$

\section{Results and Discussion}

Plotting the results for the temperature distribution for both Reynolds and Vogel viscosity models of Jayeoba and Okoya [12] who studied the flow and heat transfer under no slip and no temperature jump conditions and that of the temperature distribution obtained in this work under slip and temperature jump conditions are reported graphically below at varying parameters.

Figure 2 shows the effect of pressure drop parameter (C) for the Reynolds viscosity model on the temperature distribution. From the result, it shows that as $C$ becomes more negative for no slip condition, the maximum temperature of the fluid which is at the center of the pipe increases, while for the slip condition the maximum temperature increases but the fully developed temperature profile does not begin at the origin which was shown in Figure 3.

When heat generation term is excluded, the effect is seen as $\delta=0$. For the Reynolds viscosity model as seen in Figures 4 and 5 , as $\delta$ increases the temperature distribution of the fluid increases and the effect of the heat generation on the fluid

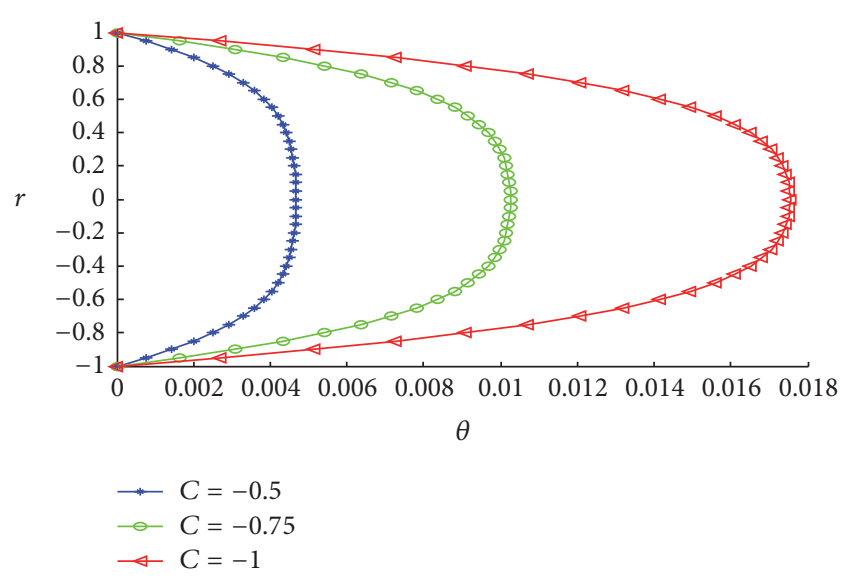

FIGURE 2: Effect of pressure gradient parameter on the temperature distribution of Reynolds viscosity model under no slip condition when $\gamma=\Gamma=\Lambda=\delta=1$.

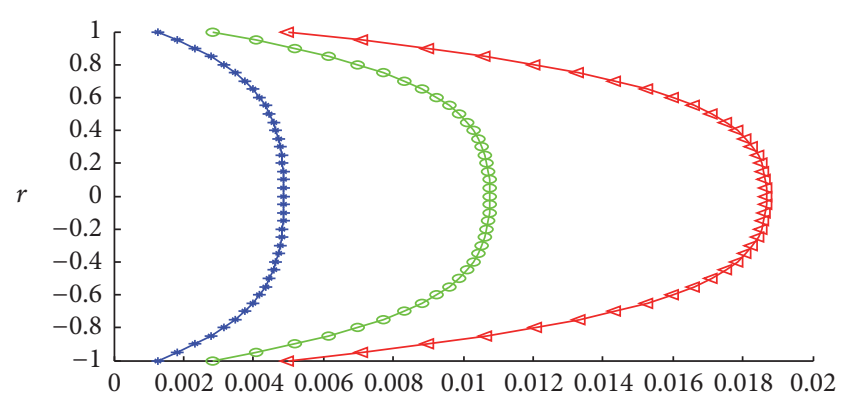

$\theta$

$$
\begin{aligned}
& \rightarrow C=-0.5 \\
& \multimap C=-0.75 \\
& \varangle C=-1
\end{aligned}
$$

FIGURE 3: Effect of varying parameter $C$ for the temperature distribution of Reynolds viscosity model under slip condition when $\Lambda=\delta=\Gamma=\gamma=1$.

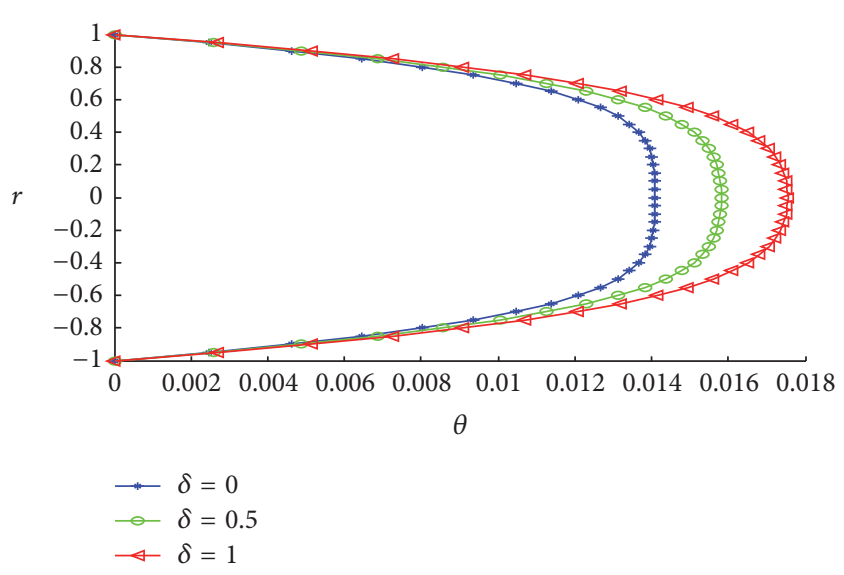

FIGURE 4: Effect of varying parameter $\delta$ for the temperature distribution of Reynolds viscosity model under no slip condition when $\Lambda=-C=\Gamma=\gamma=1$. 


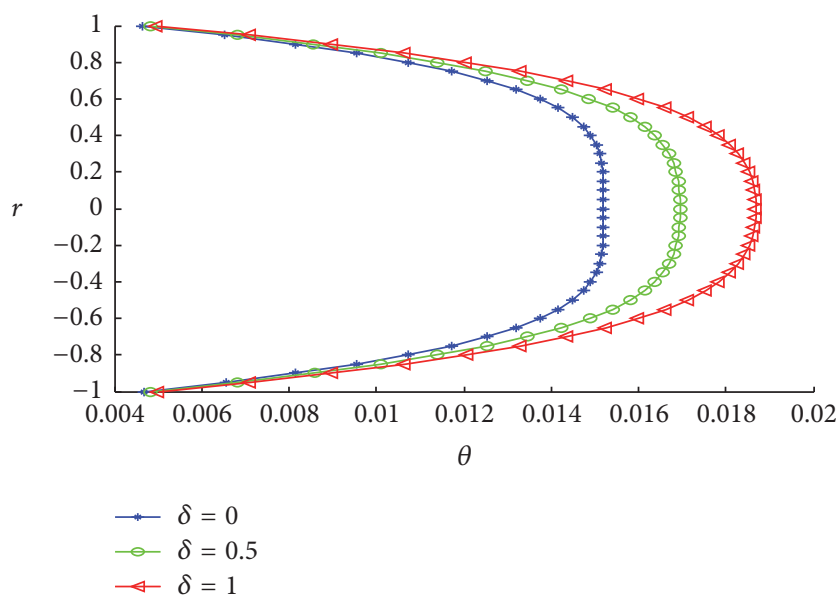

FIgURE 5: Effect of varying parameter $\delta$ for the temperature distribution of Reynolds viscosity model under slip condition when $\Lambda=-C=\Gamma=\gamma=1$.

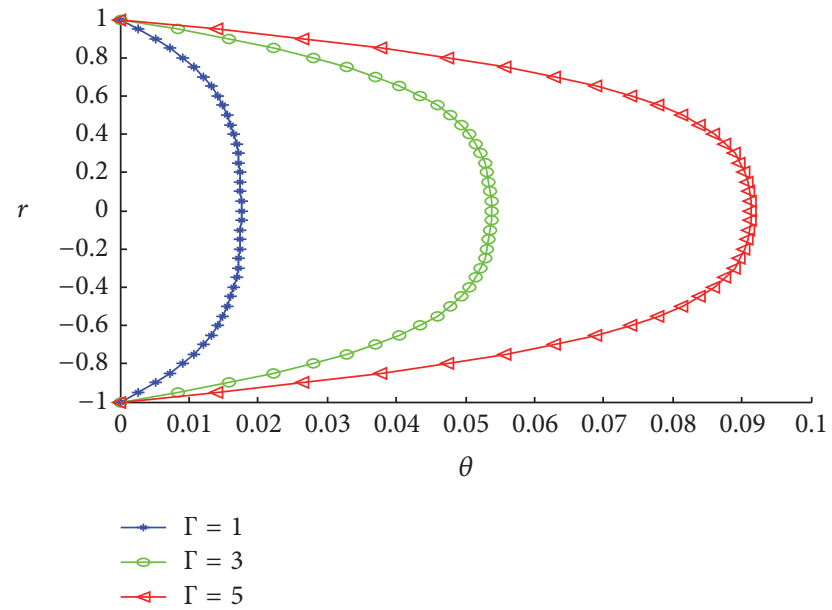

FIGURE 6: Effect of varying parameter $\Gamma$ for the temperature distribution of Reynolds viscosity model under no slip condition when $\gamma=\delta=\Lambda=-C=1$.

is maximum at the center of the pipe for no slip condition, while for slip condition the effect of increasing $\delta$ causes an increase in temperature distribution but the temperature profile does not begin at the origin. Increasing values of viscous dissipation $(\Gamma)$ gives a corresponding increase in temperature distribution for the Reynolds viscosity model because mechanical energy is converted into thermal energy. When the condition is no slip, $\Gamma$ is maximum at the pipe center as depicted in Figure 6. However, when the flow is under the slip, temperature profile is not from the origin as shown in Figure 7. The third-grade fluid can be seen to exhibit Newtonian character for the Reynolds viscosity model when $\Lambda=0$ and also the effect when the fluid behaves in non-Newtonian manner at increasing values of $\Lambda$. It can be seen from Figure 8 that, at increasing values of $\Lambda$, the temperature distribution of the fluid decreases under the no slip condition and the maximum effect of $\Lambda$ occurs at the center of the pipe, while for slip condition at increasing

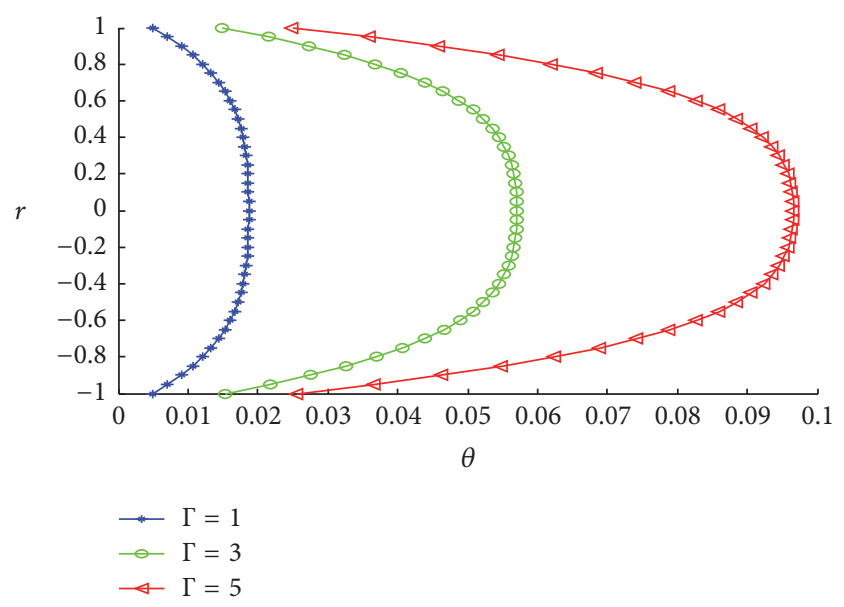

FIgURE 7: Effect of varying parameter $\Gamma$ for the temperature distribution of Reynolds viscosity model under slip condition when $\Lambda=-C=\delta=\gamma=1$.

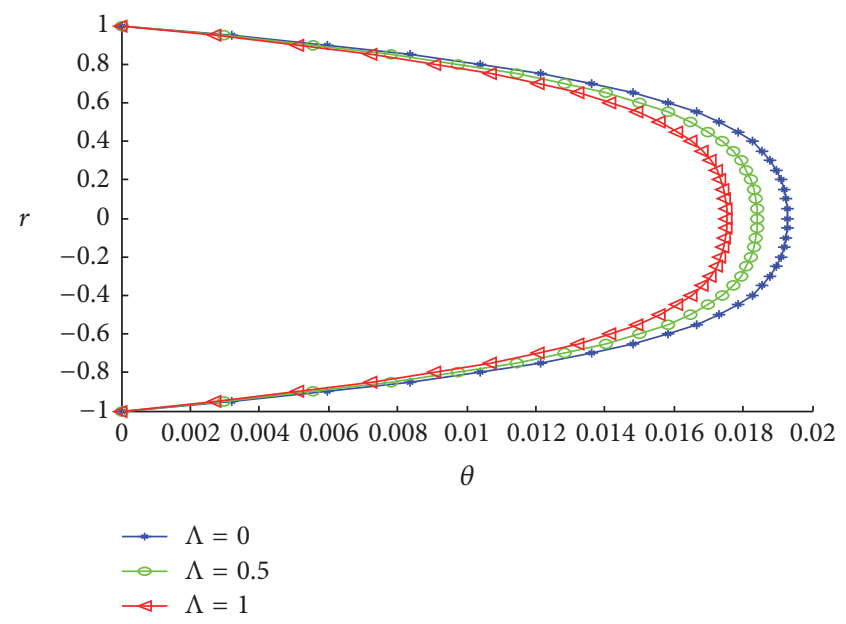

FIGURE 8: Effect of varying parameter $\Lambda$ for the temperature distribution of Reynolds viscosity model under no slip condition when $\gamma=\delta=\Gamma=-C=1$.

values of $\Lambda$ the temperature distribution decreases and the fully developed temperature profile does not begin at the origin which can be seen in Figure 9. Viscosity variation parameter $(\gamma)$ for the Reynolds viscosity model can be viewed from Figures 10 and 11. As viscosity variation parameter $(\gamma)$ increases, the temperature distribution increases for no slip condition and the effect of $\gamma$ is maximum at the center of the pipe, in Figure 10. As $\gamma$ increases for the slip condition, the temperature distribution increases and the fully developed profile does not begin at the origin as seen from Figure 11.

Increasing values of parameter $A$ in Vogel's viscosity model gives decreasing temperature distribution and the effect of Vogel's parameter $A$ is maximum at the center of the pipe for the no slip condition shown in Figure 12. But the temperature distribution profile does not begin at the origin for slip condition which can be seen in Figure 13. Also, for the Vogel viscosity parameter $B$ the influence of increasing value of $B$ shows a corresponding increase in temperature 


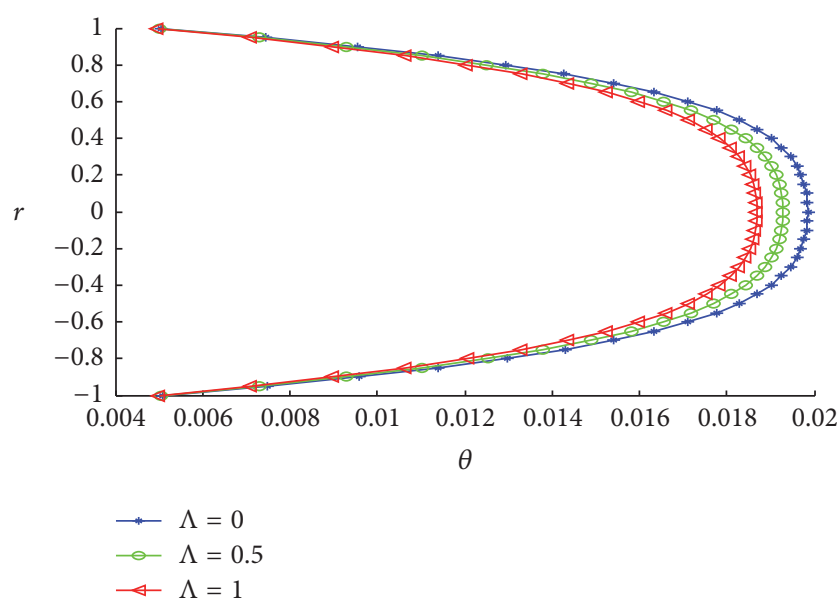

Figure 9: Effect of varying parameter $\Lambda$ for the temperature distribution of Reynolds viscosity model under slip condition when $\Gamma=-C=\delta=\gamma=1$.

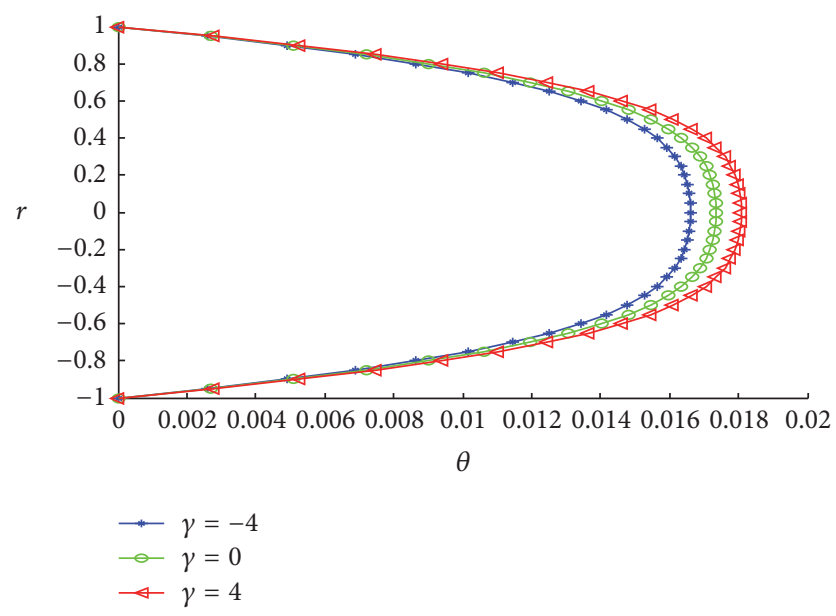

FIGURE 10: Effect of varying parameter $\gamma$ for the temperature distribution of Reynolds viscosity model under no slip condition for $\Lambda=\delta=\Gamma=-C=1$.

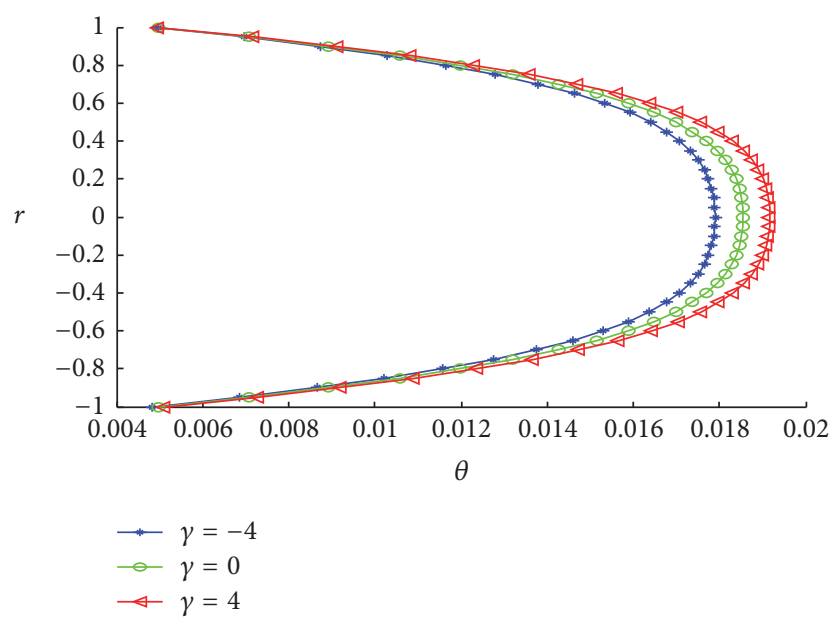

FIGURE 11: Effect of varying parameter $\gamma$ for the temperature distribution of Reynolds viscosity model under slip condition when $\Gamma=-C=\delta=\Lambda=1$.

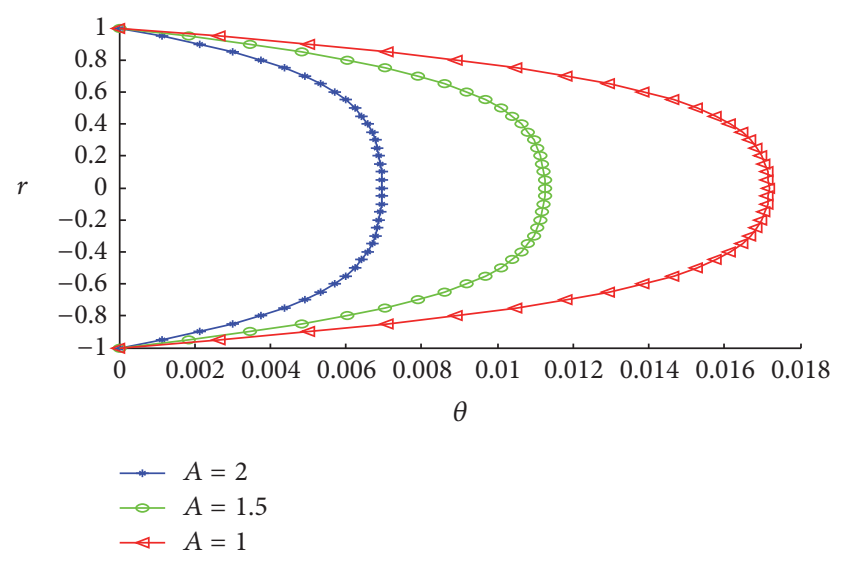

FIgURE 12: Effect of varying parameter $A$ for the temperature distribution of Vogel's viscosity model under no slip condition when $B=-C=T_{0}=\Gamma=\Lambda=\delta=1$.

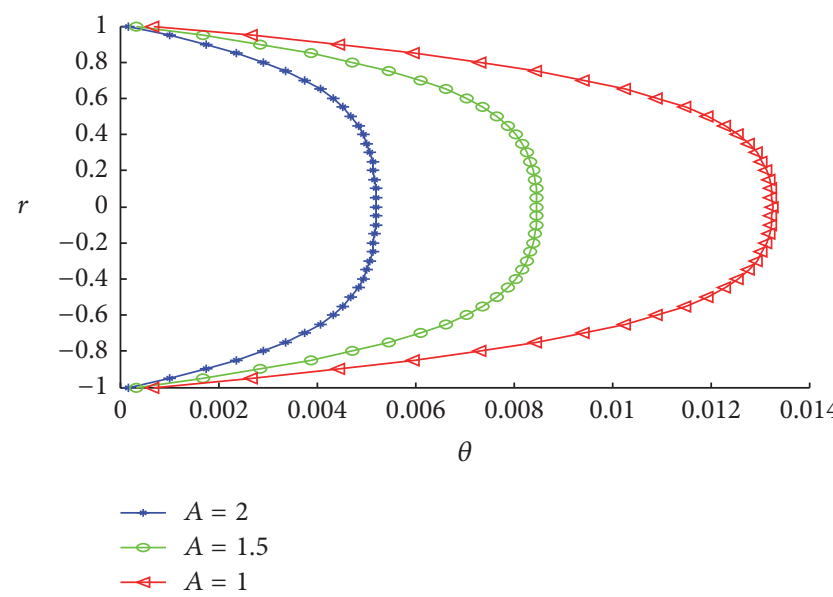

FIGURE 13: Effect of varying parameter $A$ for the temperature distribution of Vogel's viscosity model under slip condition when $B=-C=T_{0}=\Gamma=\Lambda=\delta=1$.

distribution, where the maximum temperature occurs at the center of the pipe. Fully developed temperature profile begins from the origin which can be glanced in Figure 14. And for slip condition, the temperature distribution profile is not from the origin as in Figure 15. As pressure drop parameter (C) becomes more negative for Vogel's viscosity model, the temperature distribution increases with the maximum temperature at the center of the pipe and temperature profile begins at the origin for no slip condition as shown in Figure 16, while effects of pressure drop parameter on the the temperature distribution for slip condition are shown in Figure 17. The fully developed temperature profile is not from the origin. When the heat generation term is excluded, the effect is seen as $\delta=0$ for Vogel's viscosity model. When the condition is no slip, temperature profile begins at the origin and effect of heat generation term is maximum at the center of the pipe as shown in Figure 18. And when the condition is slip, the fully developed profile does not begin at the origin, the 


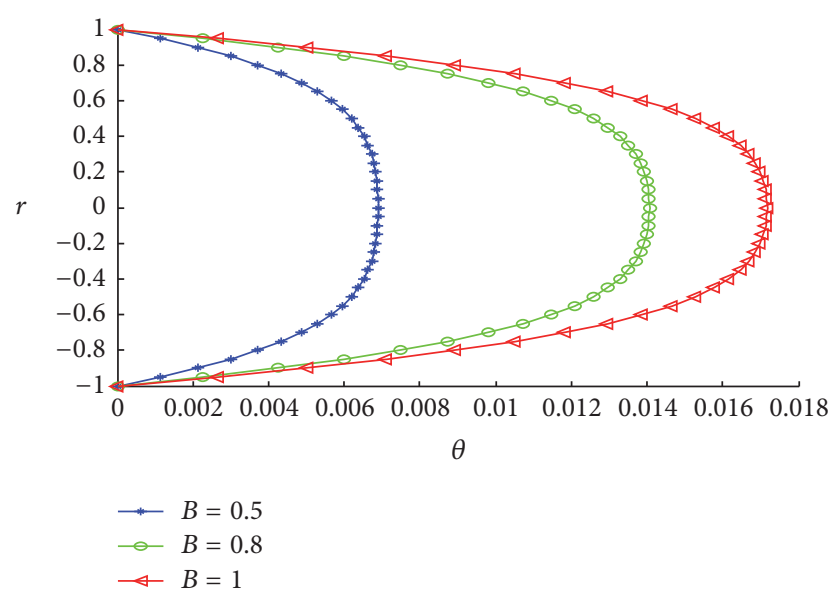

FIGURE 14: Effect of varying parameter $B$ for the temperature distribution of Vogel's viscosity model under no slip condition when $A=-C=T_{0}=\Gamma=\Lambda=\delta=1$.

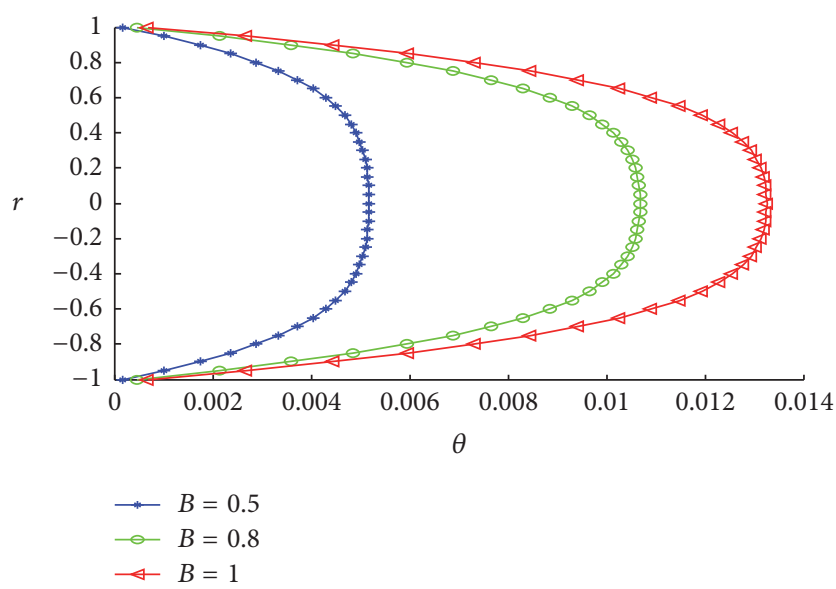

Figure 15: Effect of varying parameter $B$ for the temperature distribution of Vogel's viscosity model under slip condition when $A=-C=T_{0}=\Gamma=\Lambda=\delta=1$.

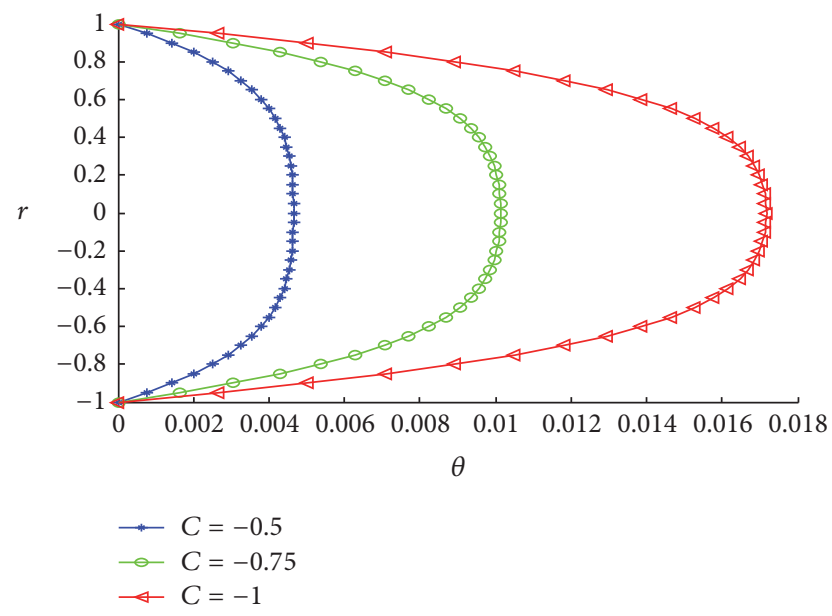

FIGURE 16: Effect of varying parameter $C$ for the temperature distribution of Vogel's viscosity model under no slip condition when $A=B=T_{0}=\Gamma=\Lambda=\delta=1$.

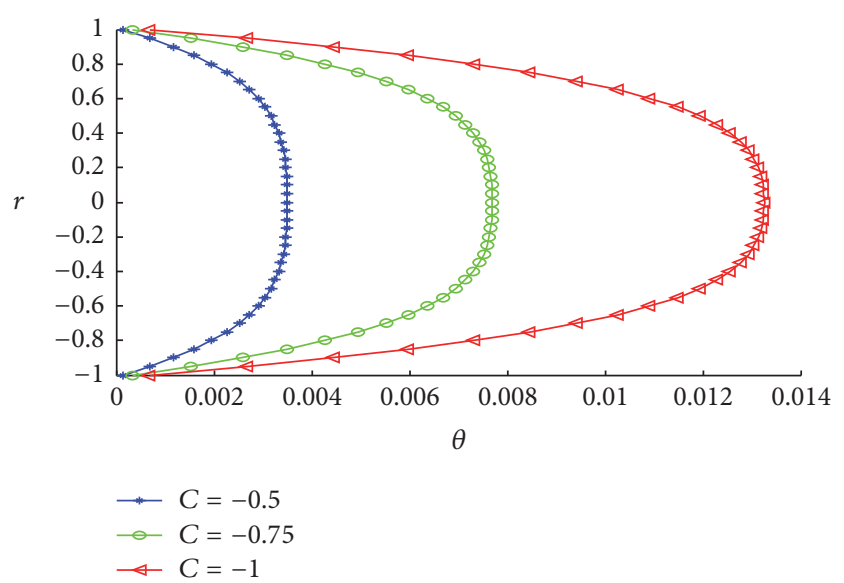

FIGURE 17: Effect of varying parameter $C$ for the temperature distribution of Vogel's viscosity model under slip condition when $A=B=T_{0}=\Gamma=\Lambda=\delta=1$.

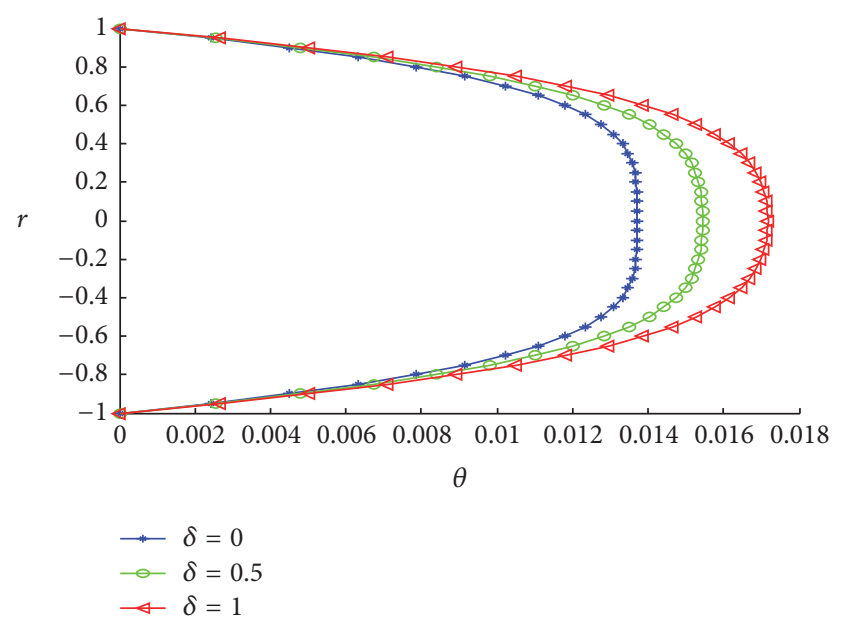

FIGURE 18: Effect of varying parameter $\delta$ for the temperature distribution of Vogel's viscosity model under no slip condition when $A=B=-C=T_{0}=\Gamma=\Lambda=1$.

effect of heat generation is also maximum at the center, and the temperature distribution increases as shown in Figure 19.

The viscous dissipation parameter $(\Gamma)$ for Vogel's viscosity model shows that, for increasing values of $\Gamma$, the temperature distribution increases. The effect of $\Gamma$ is maximum at the pipe center and the temperature profile begins at the origin for no slip condition which can be seen from Figure 20, while for slip condition the effect of $\Gamma$ is maximum at the center of the pipe and the fully developed temperature profile does not begin from the origin as shown in Figure 21. Increasing values of non-Newtonian parameter $(\Lambda)$ for Vogel's model gives decreasing values of temperature distribution where maximum effect occurs at the center of the pipe. The fluid behaves in a Newtonian manner when $\Lambda=0$, as can be seen from Figure 22. When condition is slip, the effect of increasing values of $\Lambda$ gives a corresponding decrease in 


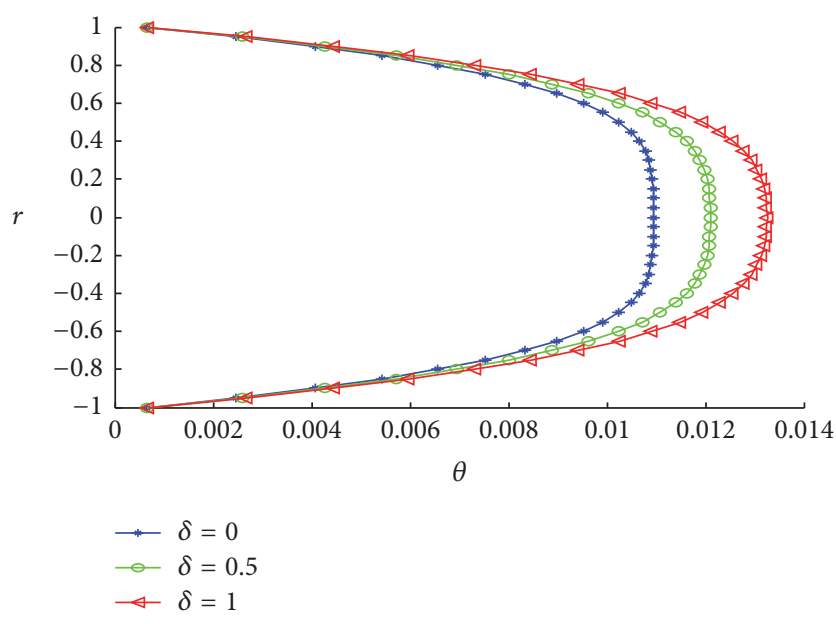

FIGURE 19: Effect of varying parameter $\delta$ for the temperature distribution of Vogel's viscosity model under slip condition when $A=B=-C=T_{0}=\Gamma=\Lambda=1$.

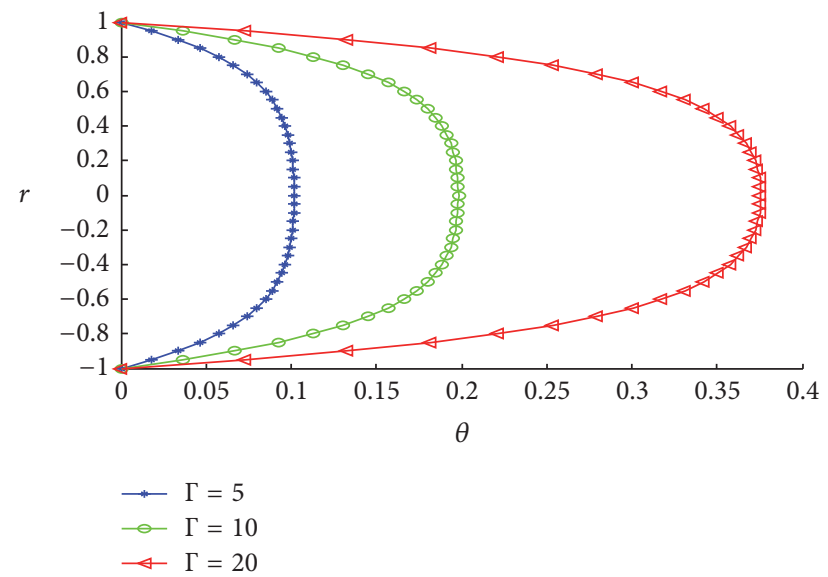

FIGURE 20: Effect of varying parameter $\Gamma$ for the temperature distribution of Vogel's viscosity model under no slip condition when $A=B=-C=T_{0}=\delta=\Lambda=1$.

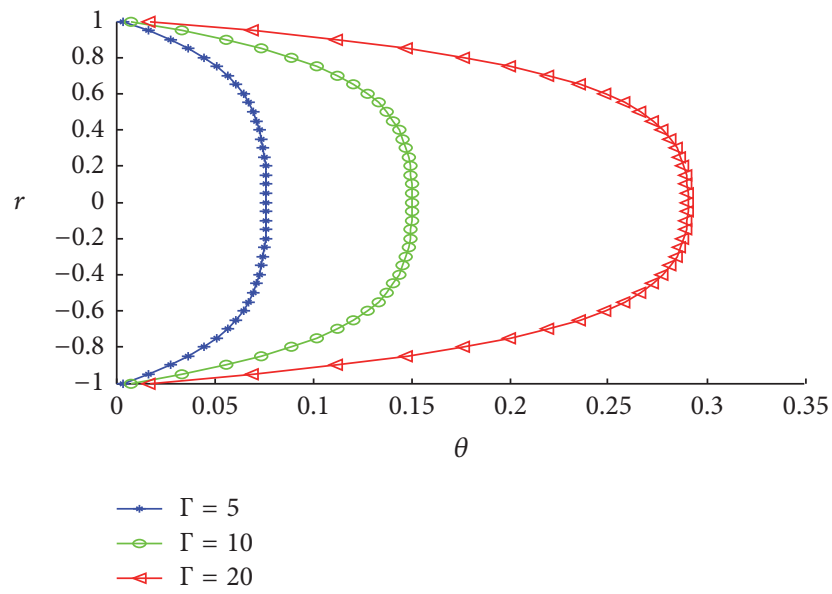

FIGURE 21: Effect of varying parameter $\Gamma$ for the temperature distribution of Vogel's viscosity model under slip condition when for $A=B=-C=T_{0}=\delta=\Lambda=1$.

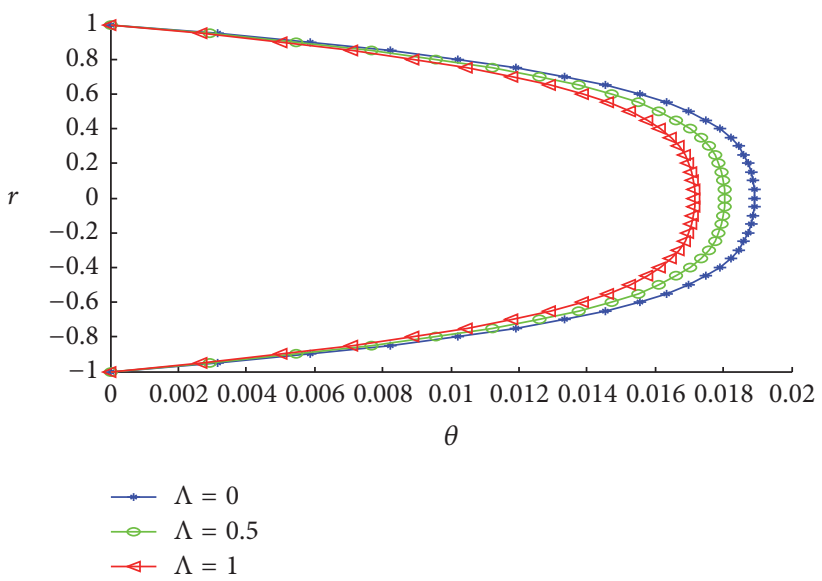

FIGURE 22: Effect of varying parameter $\Lambda$ for the temperature distribution of Vogel's viscosity model under no slip condition when $A=B=-C=T_{0}=\delta=\Gamma=1$.

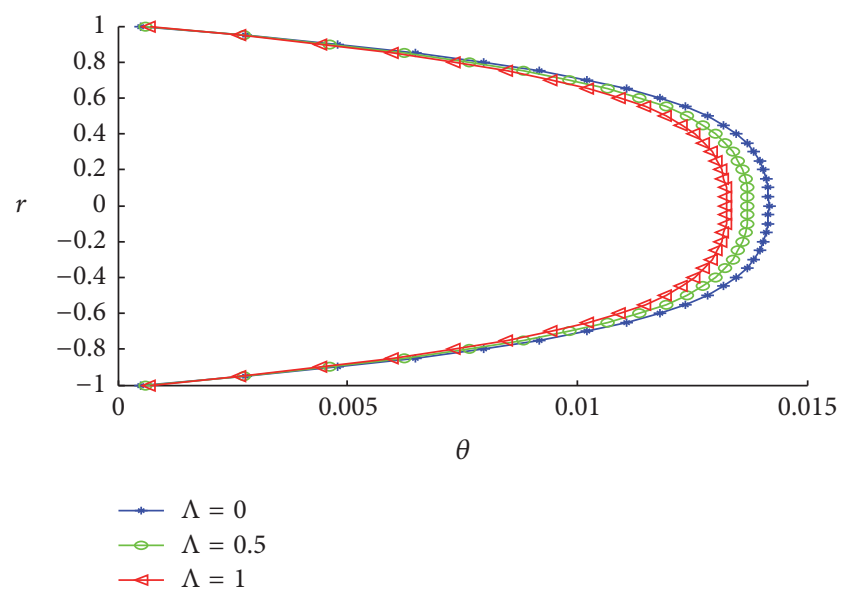

FIgURE 23: Effect of varying parameter $\Lambda$ for the temperature distribution of Vogel's viscosity model under slip condition when for $A=B=-C=T_{0}=\delta=\Gamma=1$.

temperature distribution and the fully developed temperature profile does not begin at the origin which can be seen in Figure 23. When the initial temperature term $T_{0}$ for Vogel's viscosity model is increased, there is a corresponding increase in temperature distribution. The effect of $T_{0}$ is maximum at the center of the pipe. When condition is no slip, the fully developed temperature profile begins from the origin as shown in Figure 24 and for slip condition increase in $T_{0}$ gives a corresponding increase in temperature distribution with the fully developed temperature profile not beginning from the origin, as depicted in Figure 25. From the velocity distribution in Figure 26, as the Knudsen number increases from zero, it can be seen that the velocity profile reduces, with the arrow indicating the direction of increasing Knudsen number. Thus, as the Knudsen number increases, the profile of the velocity decreases. 


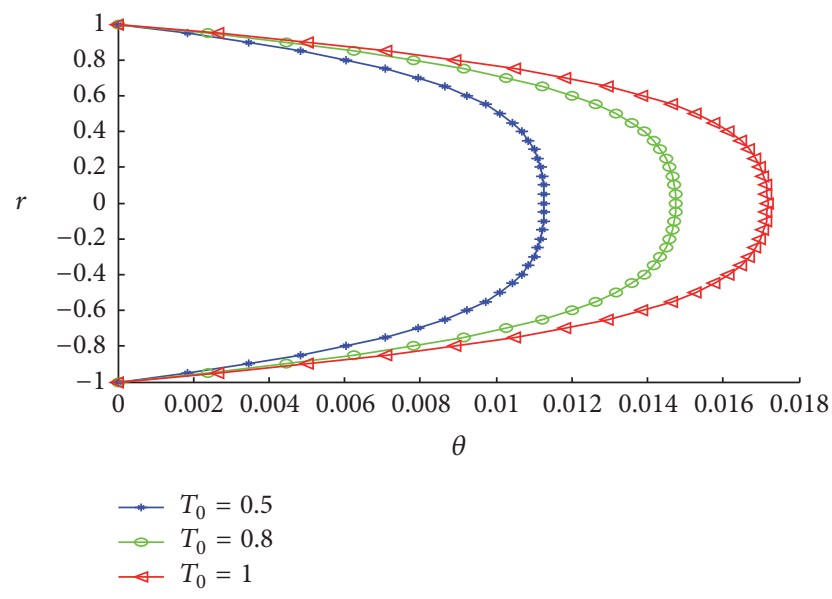

FIGURE 24: Effect of varying parameter $T_{0}$ for the temperature distribution of Vogel's viscosity model under no slip condition when $B=-C=\Lambda=\delta=\Gamma=1$.

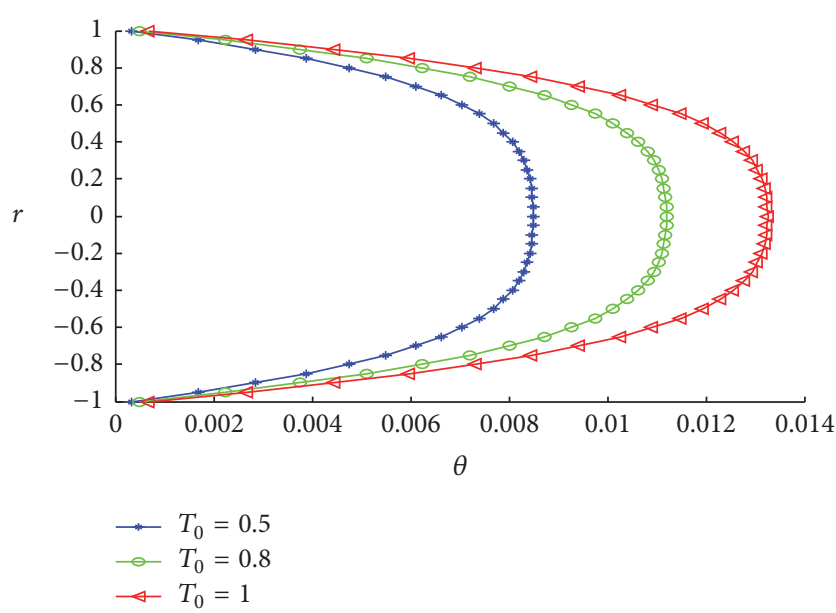

FIGURE 25: Effect of varying parameter $T_{0}$ for the temperature distribution of Vogel's viscosity model under slip condition when $A=B=-C=\Lambda=\delta=\Gamma=1$.

\section{Conclusion}

In this work, approximate analytical solutions for the nonlinear analysis of heat transfer in a pipe flow of a thirdgrade fluid with temperature-dependent viscosities and heat generation under slip and no slip conditions have been developed using regular perturbation technique. The obtained analytical solutions were used to investigate the effects of slip boundary conditions on the heat transfer in the pipe flow. Also, the effects of parameters such as variable viscosity, non-Newtonian parameter, viscous dissipation, and pressure gradient at various values (which indicate different fluid flow situations) are established. The results can be used to advance the analysis and study of the behavior of third-grade fluid flow and steady state heat transfer processes such as those found in coal slurries, polymer solutions, textiles, ceramics, catalytic reactors, and oil recovery applications.

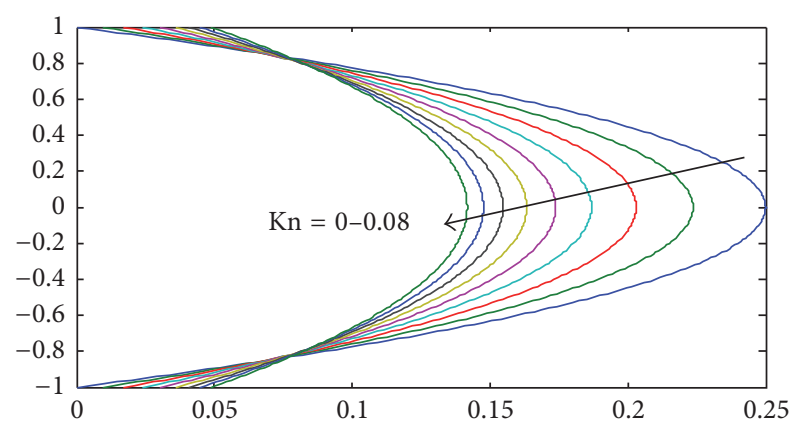

FIGURE 26: Velocity distribution profile as the Knudsen number increases from 0 to 0.08 .

\section{Nomenclature}

$A$ and $B$ :

$M:$

$A=a /\left(\overline{T_{0}} \beta\right):$
$B=\left(b+\overline{T_{0}}\right) /\left(\overline{T_{0}} \beta\right):$
$C_{0}:$
$C=\left(R^{2} / \mu_{0}^{e} w_{0}\right)(\partial P / \partial Z):$
$K:$
$\partial P / \partial r:$
$\partial P / \partial Z:$
$\partial P / \partial \phi:$
$Q:$
$r:$

$\bar{r}=r / R$ :

$\frac{R:}{T_{0}}:$

$W(r)$ :

$\bar{w}=w / w_{0}:$

$w_{0}:$

$z$ :

$\alpha_{1}, \alpha_{2}, \alpha_{3}$ :

$\beta=R \overline{T_{0}} / E$ :

$\gamma=M \beta \overline{T_{0}}$ :

$\mu:$

$\bar{\mu}=\mu / \mu_{0}^{e}:$

$\Gamma=4 \mu_{0}^{e} w_{0}^{2} /\left(K \overline{T_{0}} \beta\right):$

$\theta=\left(T-T_{0}\right) E /\left(R \overline{T_{0}^{2}}\right)$ :

$\Lambda=\beta_{3} w_{0}^{2} /\left(\mu_{0}^{e} r_{0}^{2}\right):$

$\delta=Q E A_{0} R^{2} C_{0} /\left(R K \overline{T_{0}^{2}}\right)$
Constants from dimensional Vogel's viscosity model Constant from dimensional Reynolds viscosity model Dimensionless constant from Vogel's viscosity model

Dimensionless constant from Vogel's viscosity model Initial concentration of reacting species

Pressure gradient parameter Constant thermal conductivity Pressure gradient along the normal to the pipe axis Pressure gradient in the axial direction

Pressure gradient in rotational direction

Heat generation constant Dimensional perpendicular distance in the pipe axis Dimensionless perpendicular distance in the pipe axis Radius of the pipe Initial temperature Dimensional velocity component in the $z$-axis Dimensionless velocity component in the $z$-axis Dimensional reference velocity Axis of the cylinder Material constants Activation energy parameter Reynolds viscosity variational parameter

Dynamic shear viscosity Dimensionless viscosity Viscous heating parameter

Dimensionless temperature excess Non-Newtonian material parameter of the fluid : Heat generation parameter. 


\section{Competing Interests}

The authors declare that there are no competing interests regarding the publication of this paper.

\section{References}

[1] R. L. Fosdick and K. R. Rajagopal, "Thermodynamics and stability of fluids of third grade," Procter Society London, vol. 339, pp. 351-377, 1980.

[2] S. N. Majhi and V. R. Nair, "Flow of a third grade fluid over a sternosed tubes," Indian National Science Academy, vol. 60, no. 3, p. 535, 1994.

[3] M. Massoudi and I. Christie, "Effects of variable viscosity and viscous dissipation on the flow of a third grade fluid in a pipe," International Journal of Non-Linear Mechanics, vol. 30, no. 5, pp. 687-699, 1995.

[4] M. Yürüsoy and M. Pakdemirli, "Approximate analytical solutions for the flow of a third-grade fluid in a pipe," International Journal of Non-Linear Mechanics, vol. 37, no. 2, pp. 187-195, 2002.

[5] K. Vajravelu, J. R. Cannon, D. Rollins, and J. Leto, "On solutions of some non-linear differential equations arising in third grade fluid flows," International Journal of Engineering Science, vol. 40, no. 16, pp. 1791-1805, 2002.

[6] T. Hayat, S. Nadeem, S. Asghar, and A. M. Siddiqui, "Fluctuating flow of a third-grade fluid on a porous plate in a rotating medium," International Journal of Non-Linear Mechanics, vol. 36, no. 6, pp. 901-916, 2001.

[7] Y. Muhammet, "Similarity solutions to boundary layer equations for third grade non-Newtonian fluid in special channel coordinate system," Journal of Theoretical and Applied Mechanics, vol. 41, no. 4, pp. 775-787, 2003.

[8] M. Yürüsoy, "Flow of a third grade fluid between concentric circular cylinders," Mathematical and Computational Applications, vol. 9, no. 1, pp. 11-17, 2004

[9] M. Pakdemirli and B. S. Yilbas, "Entropy generation for pipe flow of a third grade fluid with Vogel model viscosity," International Journal of Non-Linear Mechanics, vol. 41, no. 3, pp. 432437, 2006.

[10] M. Sajid, R. Mahmood, and T. Hayat, "Finite element solution for flow of a third grade fluid past a horizontal porous plate with partial slip," Computers \& Mathematics with Applications, vol. 56, no. 5, pp. 1236-1244, 2008.

[11] R. Ellahi, T. Hayat, F. M. Mahomed, and S. Asghar, "Effects of slip on the non-linear flows of a third grade fluid," Nonlinear Analysis: Real World Applications, vol. 11, no. 1, pp. 139-146, 2010.

[12] O. J. Jayeoba and S. S. Okoya, "Approximate analytical solutions for pipe flow of a third grade fluid with variable model of viscosities and heat generation/absorption," Journal of the Nigerian Mathematical Society, vol. 31, pp. 207-227, 2012.

[13] S. S. Abbasbandy, T. Hayat, R. Ellahi, and S. Asghar, "Numerical results of a flow in a third grade fluid between two porous walls," Zeitschrift für Naturforschung A, vol. 64, no. 1-2, pp. 59-64, 2009.

[14] K. S. Adegbie and F. I. Alao, "Flow of a newtonian fluid in a symmetrically heated channel: effect of viscosity and viscous dissipation," Mathematical Problems in Engineering, vol. 2006, Article ID 29314, 7 pages, 2006.

[15] Y. M. Aiyesimi, G. T. Okedayo, and O. W. Lawal, "Unsteady magnetohydrodynamic (MHD) thin film flow of a third grade fluid with heat transfer and no slip boundary condition down an inclined plane," International Journal of Physical Sciences, vol. 8, no. 19, pp. 946-955, 2013.
[16] R. P. Chhabra, Non Newtonian Fluid: An Introduction, Indian Institute of Technology, New Delhi, India, 2010.

[17] R. P. Chhbra and J. F. Richardson, Non Newtonian Flow and Rheology, Butterworth-Heinemann, Oxford, UK, 2nd edition, 1999.

[18] R. Ellahi, M. Raza, and K. Vafai, "Series solutions of nonNewtonian nanofluids with Reynolds' model and Vogel's model by means of the homotopy analysis method," Mathematical and Computer Modelling, vol. 55, no. 7-8, pp. 1876-1891, 2012.

[19] T. Hayat, Z. Iqbal, M. Mustafa, and A. A. Hendi, "Melting heat transfer in the stagnation-point flow of third grade fluid past a stretching sheet with viscous dissipation," Thermal Science, vol. 17, no. 3, pp. 865-875, 2013.

[20] T. Hayat, A. H. Kara, and E. Momoniat, "The unsteady flow of a fourth-grade fluid past a porous plate," Mathematical and Computer Modelling, vol. 41, no. 11-12, pp. 1347-1353, 2005.

[21] M. Kothandapani, "The influence of exponential viscosity on a MHD viscoelastic fluid over a stretching sheet," International Journal of Applied Mathematics and Mechanics, vol. 9, no. 5, p. 81, 2012.

[22] S. Nadeem and M. Ali, "Analytical solutions for pipe flow of a fourth grade fluid with Reynolds and Vogel's model of viscosities," Communications in Nonlinear Mechanics, vol. 43, pp. 558-599, 2009.

[23] S. Nadeem and H. Sadia, "Influence of temperature dependent viscosity and entropy generation on the flow of a JohnsonSegalman fluid," Walailak Journal, vol. 10, no. 5, pp. 553-557, 2013. 


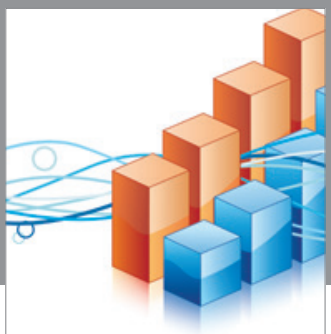

Advances in

Operations Research

vatem alat4

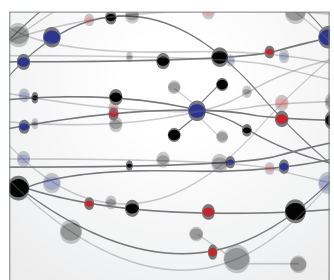

\section{The Scientific} World Journal
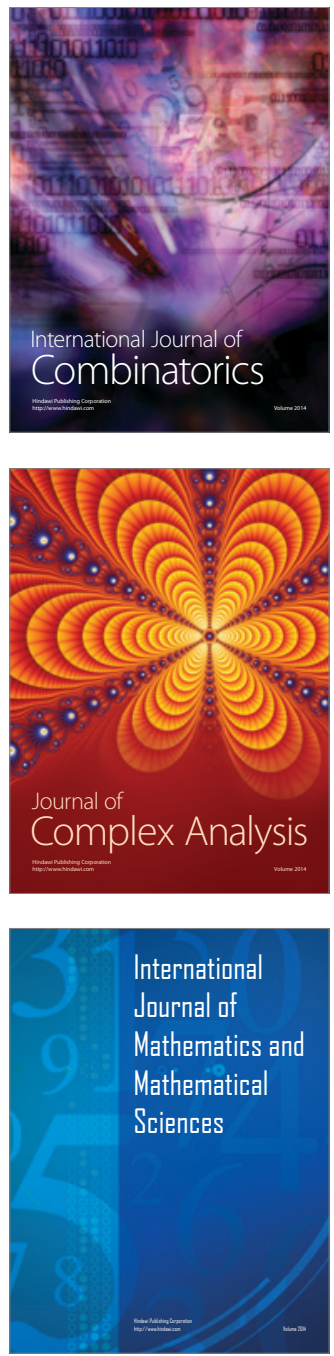
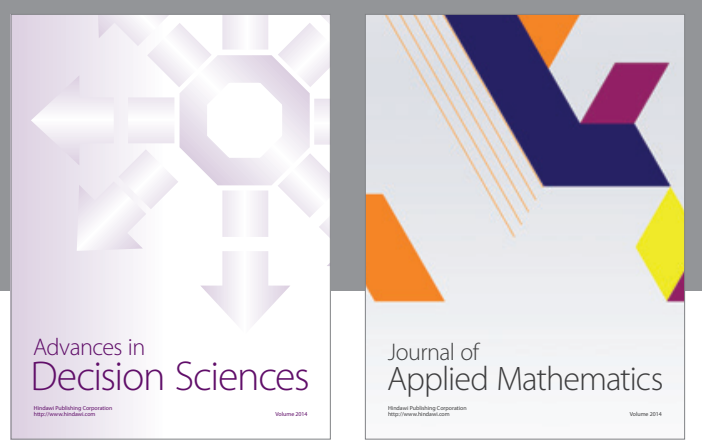

Algebra

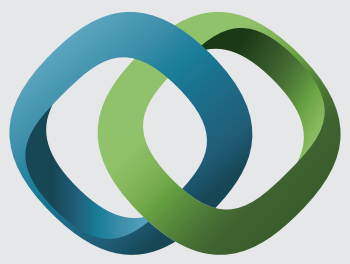

\section{Hindawi}

Submit your manuscripts at

http://www.hindawi.com
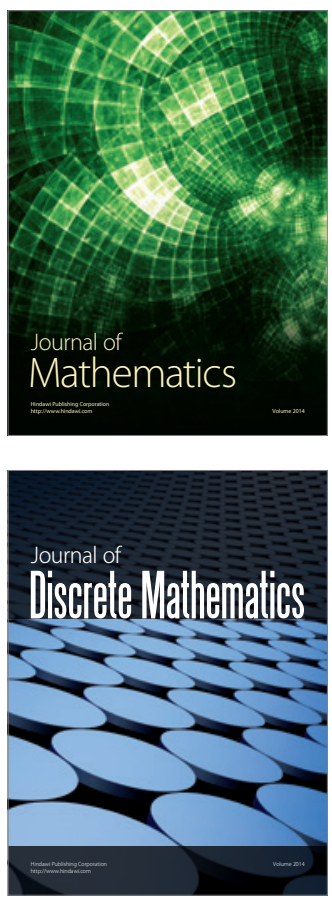

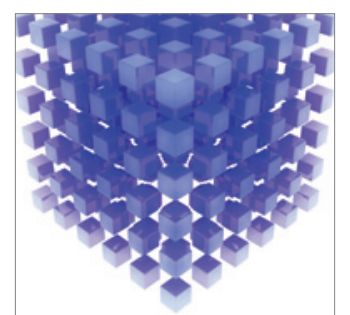

Mathematical Problems in Engineering
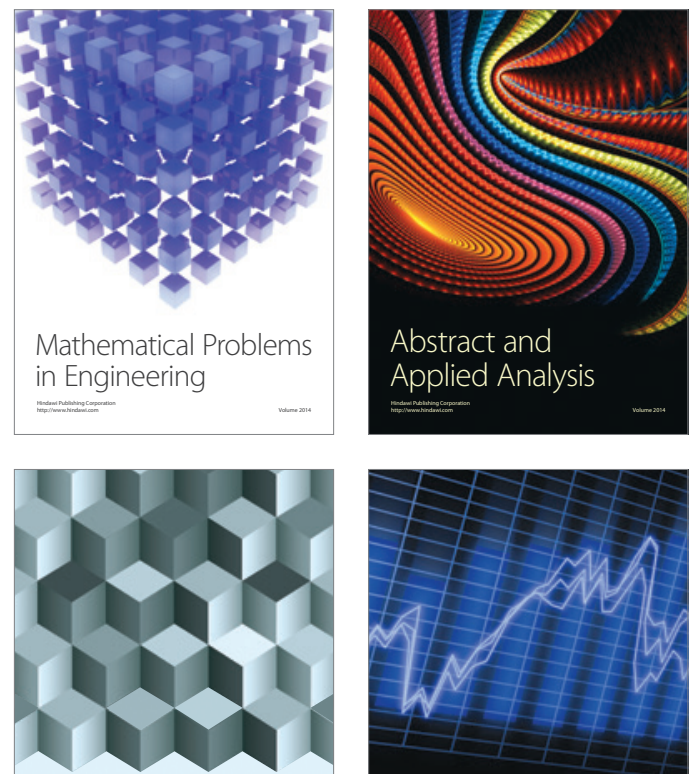

Journal of

Function Spaces

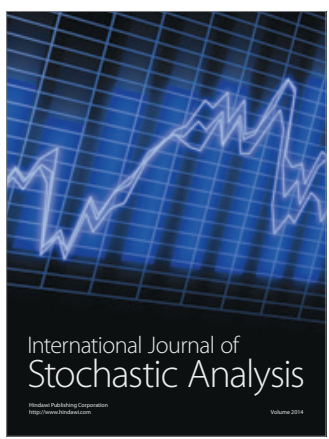

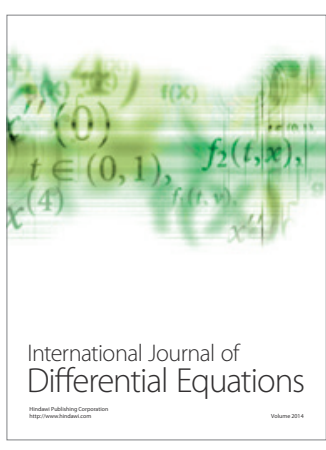
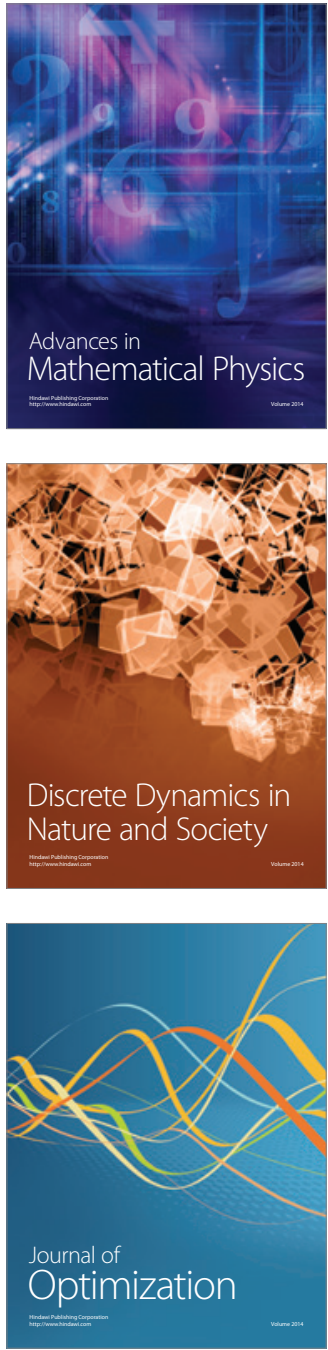